\title{
Investigating Effects of the Ball Size on the Gas Throughput Using Partially Curved and Wholly Curved Seats
}

\author{
Saleh Ahmed ${ }^{1 *}$, Fathi Elldakli ${ }^{2}$, Lloyd Heinze ${ }^{1}$, Khalid Elwegaa ${ }^{1}$, Hossein Emadi ${ }^{1}$ \\ ${ }^{I}$ Bob L. Herd Department of Petroleum Engineering, Texas Tech University, Lubbock, TX 79409, USA \\ ${ }^{2}$ Department of Petroleum Engineering, University of Wyoming, Laramie, WY 82071, USA
}

*Corresponding Author: Saleh Ahmed, Bob L. Herd Department of Petroleum Engineering, Texas Tech University, Lubbock, TX 79409, USA

\begin{abstract}
Gas lift is considered the most important artificial method in the oil and gas industry. Although, there are several components in the Gas Lift Valve (GLV), the ball and the seats are the only two adjustable components. The objective of this study is to maximize the gas throughput using the new design seats of partially curved design (PCD) and wholly curved design (WCD) to increase oil production.
\end{abstract}

Five main seat designs were evaluated using Computational Fluid Dynamics (CFD): The Sharp Edge, modified design, the optimized design, Partially Curved Design and Wholly Curved Design. The modified design and the optimized design have been previously evaluated. However, PCD and WCD are evaluated in this research. To maximize the gas throughput, seven seat sizes and five different ball sizes have been selected in this simulation study. Each seat has Port Bottom Diameter (PBD) and Port Top Diameter (PTD). The criterion of the best case is to have the highest gas throughput.

The CFD results showed that PCD has $11 \%$ higher gas throughput than the modified design at the ball size 6/16 inches, PBD 5/16 inches and PTD 7/16 inches. Also, WCD has 6\% higher gas throughput than the optimized design at the ball size 7/16 inches, PBD 6/16 inches and PTD 8/16 inches. The results also demonstrated that the best design seat is the Wholly Curved Design compared to the others when the PBD, $P T D$ and stem travel are 5/16 in, 7/16 in and 5/16 in, respectively resulting in the highest gas flow rate of $0.65 \mathrm{~kg} / \mathrm{s}$. The novelty of this research is describing four different GLV designs, two of which were created. Additionally, the results show the effect of each of the adjustable GLV components: PBD, PTD, and stem travel.

Keywords: Artificial Gas Lift, Gas Lift Valve, Ball Configuration, Seat Size, Gas Throughput.

\section{INTRODUCTION}

Gas lift is considered as one of the artificial lift methods, which has been applied extensively for several decades and used by $10 \%$ of the artificial lift methods in oil industry. Carl Loscher, 1797 initially conducted laboratory experiments using compressed air to lift liquids. This technique was later used to lift water from pit swing. In this technique, air was injected into liquid at the bottom of tubing through a valve. Later, Airlift was used in the oil industry to obtain oil from wells and it was known as the gas lift method (John W. Booth 1864). An American engineer named Cockford used an air-filled pipe connected to the tubing to decrease hydrostatic pressure by reducing oil density, allowing the well to produce more. In the 1920's, for safety reasons, air was replaced by natural gas to be used to lift oil and this application was successfully applied in the Seminole field in Oklahoma.

In the 1930's, Spring-operated differential GLV was invented. The valve operates only when the injected pressure (casing pressure) is greater than tubing pressure. Brown,1984 developed another GLVs model, which mechanically operated from the surface, using tubing string. Wireline retrievable GLVs were introduced instead of the mechanical one due to the difficulty and unreliability of tubing retrievable GLVs.

(Winkler and Blann, 2007) defined the gas lift as a simple and flexible artificial lift method in which external gas is injected continuously or intermittently from the casing-tubing annulus into the tubing string through specially designed valves (GLV). Although sophisticated gas lift technologies such as smart GLV (Xu et al., 2013) and advanced Gas-Lift Insert System (GLIS) (Aliyeva and Novruzaliyev, 

Seats

2015) have been developed, all GLVs are still being manufactured based on King 's design (King, 1940). This design consists of a nitrogen-charged dome section and bellows assembly. Benchmark GLV is another type of GLV in which the nitrogen-charged dome and bellows assembly are absent. Laboratory testing is the primary use of this type of GLV. However, Winkler and Camp (1987) successfully used benchmark valve in field applications.

A GLV basically regulates the pressure on its upstream side on its downstream. GLV remains open despite that the upstream pressure is higher than the dome-charged pressure. Therefore, calibrating the GLV is important to the artificial lift cycle for each well to achieve the best performance of the well. The two main adjustable compartments of the GLV are the ball and the seat.

The objective of this study is to optimize the GLV performance by measuring the dynamic gas throughput of each GLV using the new design seats of Partially curved and Wholly curved developed by Ansys 19. Computational Fluid Dynamics technique (CFD) is used to simulate the flow behavior through GLV and to develop an in depth understanding of pressure distribution and fluid flow inside the valve. The results of the simulation work of the new designs were compared to the previous designs (Sharp-Edge, Modified and optimized designs).

\section{Simulation WORK}

\subsection{Computational Fluid Dynamics Technique (CFD)}

Computational Fluid Dynamics technique (CFD) is used to simulate the flow behavior through GLV and to develop an in depth understanding of pressure distribution and fluid flow inside the valve. The results of the simulation work of the new designs were compared to the previous designs (Sharp-Edge, Modified and optimized designs). CFD technique converts differential Navier-Stockes equations to a system of algebraic equations representing the flow at those points. Since the problem is too complex to be solved analytically, a numerical simulator is used. Figure 1 shows systematic steps toward building the CFD simulation model.

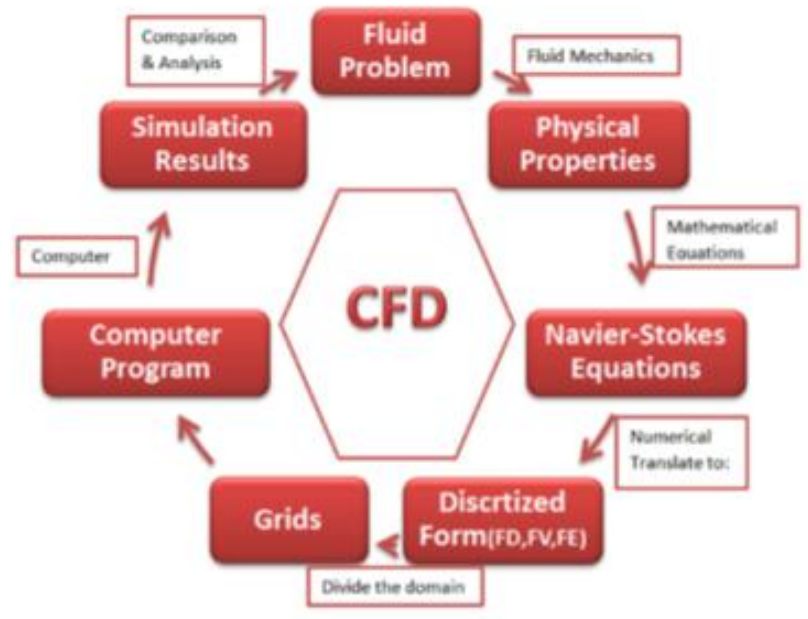

Figure1. Shows systematic steps toward building the CFD simulation model. (Elldakli 2017)

There are three processes to simulate the flow behavior using a CFD simulation model as follows:

- Pre-processor phase: it is used to build the geometry domain of the section.

- Solver: it integrates the governing equations, fluid flow, and the boundary equations and solve the algebraic equations using iterative methods.

- Post-processor phase: it enables the end-user to display the results graphically, which include the domain geometry, grid display, and the vector plots

\subsection{Case Study}

Despite the successful recent seat modifications of gas lift valves (Sharp edge, Modified and Optimized seats), it is believed that new seat designs are needed to increase the oil production by increasing the gas flow rate thorough the valve. 

Seats

The two new design seats as shown in figure 2:

1. Partially curved design seat

2. Wholly curved design seat

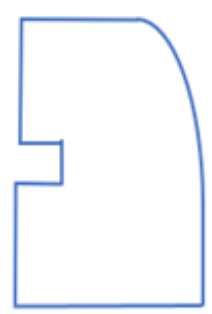

A

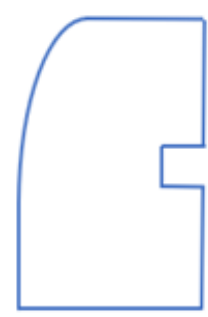

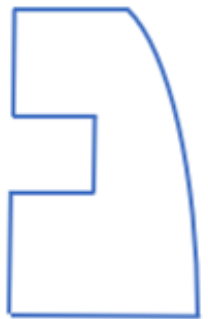

B

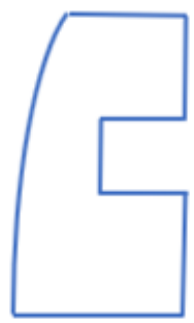

Figure2. A) Partially Curved Design Seat, B) Wholly Curved Design Seat

Using Ansys 19 Software to simulate the two new designs by first modelling the pressure distribution inside the gas lift valve. In order to do that, the following values of inlet and outlet pressures of boundary conditions were entered $(600 \mathrm{psi}$ and $0.1 \mathrm{psi})$ respectively, then the results of the new designs were compared with the previous design seats.

Figure3. Shows the case study seat and ball size which was modeled by the Fluent Ansys 19 software.

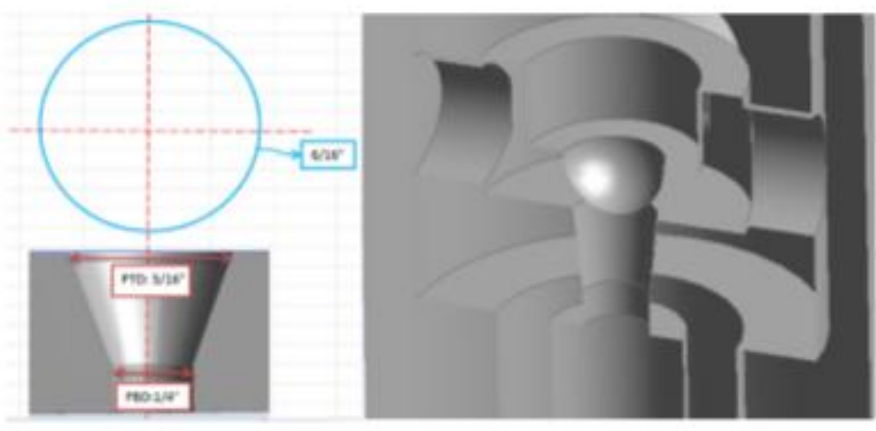

Figure3. Model case (Seat and Ball), (Elldakli 2017)

Figure4. Presents the laboratory set up and the encapsulating part that was simulated using ANSYS software

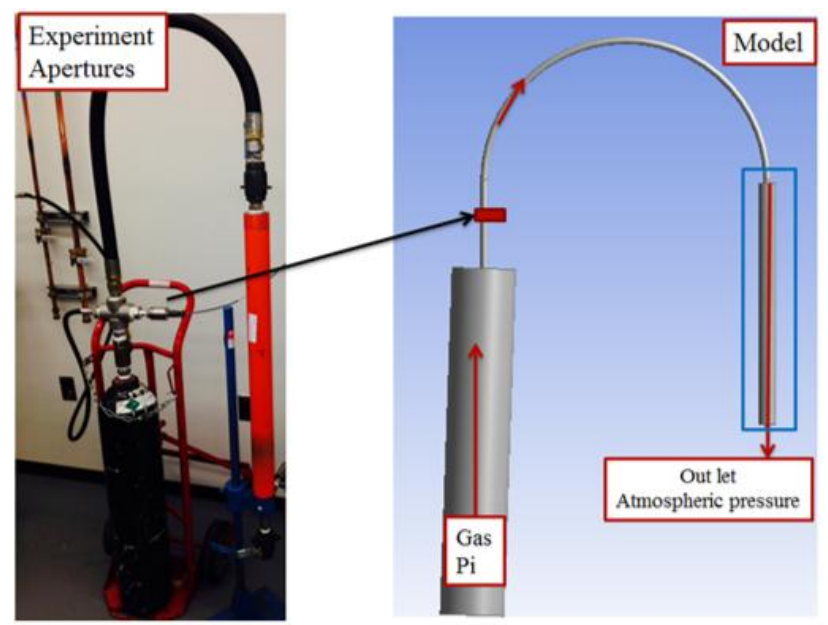

Figure4. The setup modeled parts, (Elldakli 2017)

\subsubsection{Ball Size and Design Seat Scenarios}

Different scenarios were used in this study taking into account the ball sizes, PBD and PTD. Five different ball sizes and seven seats were used to simulate the gas throughput. The following tables represent all the scenarios that were conducted: 
Investigating Effects of the Ball Size on the Gas Throughput Using Partially Curved and Wholly Curved Seats

Table1. Case no. 1: PBD 4/16 in and PTD 6/16 in at different ball sizes

\begin{tabular}{|c|c|c|c|c|}
\hline PBD, in & PTD, in & Ball, in & stem, in & Y, coordinate \\
\hline $4 / 16$ & $6 / 16$ & $5 / 16$ & 0.414 & 0.2773 \\
\hline $4 / 16$ & $6 / 16$ & $6 / 16$ & 0.288 & 0.2690 \\
\hline $4 / 16$ & $6 / 16$ & $7 / 16$ & 0.074 & 0.1867 \\
\hline $4 / 16$ & $6 / 16$ & $8 / 16$ & 0.063 & 0.2284 \\
\hline
\end{tabular}

Table2. Case no. 2: PBD 4/16 in and PTD 7/16 in at different ball sizes

\begin{tabular}{|c|c|c|c|c|}
\hline PBD, in & PTD, in & Ball, in & stem, in & Y, coordinate \\
\hline $4 / 16$ & $7 / 16$ & $5 / 16$ & 0.392 & 0.2121 \\
\hline $4 / 16$ & $7 / 16$ & $6 / 16$ & 0.321 & 0.2475 \\
\hline $4 / 16$ & $7 / 16$ & $7 / 16$ & 0.251 & 0.2181 \\
\hline $4 / 16$ & $7 / 16$ & $8 / 16$ & 0.067 & 0.1880 \\
\hline
\end{tabular}

Table3. Case no. 3: PBD 4/16 in and PTD 8/16 in at different ball sizes

\begin{tabular}{|c|c|c|c|c|}
\hline PBD, in & PTD, in & Ball, in & stem, in & Y, coordinate \\
\hline $4 / 16$ & $8 / 16$ & $5 / 16$ & 0.33 & 0.132 \\
\hline $4 / 16$ & $8 / 16$ & $6 / 16$ & 0.289 & 0.1824 \\
\hline $4 / 16$ & $8 / 16$ & $7 / 16$ & 0.260 & 0.2268 \\
\hline $4 / 16$ & $8 / 16$ & $8 / 16$ & 0.219 & 0.1698 \\
\hline $4 / 16$ & $8 / 16$ & $9 / 16$ & 0.0614 & 0.1902 \\
\hline
\end{tabular}

Table4. Case no. 4: PBD 5/16 in and PTD 7/16 in at different ball sizes

\begin{tabular}{|c|c|c|c|c|}
\hline PBD, in & PTD, in & Ball, in & stem, in & Y, coordinate \\
\hline $5 / 16$ & $7 / 16$ & $5 / 16$ & 0.636 & 0.3468 \\
\hline $5 / 16$ & $7 / 16$ & $6 / 16$ & 0.508 & 0.3745 \\
\hline $5 / 16$ & $7 / 16$ & $7 / 16$ & 0.38 & 0.3578 \\
\hline $5 / 16$ & $7 / 16$ & $8 / 16$ & 0.1014 & 0.2224 \\
\hline $5 / 16$ & $7 / 16$ & $9 / 16$ & 0.0868 & 0.2636 \\
\hline
\end{tabular}

Table5. Case no. 5: PBD 5/16 in and PTD 8/16 in at different ball sizes

\begin{tabular}{|c|c|c|c|c|}
\hline PBD, in & PTD, in & Ball, in & stem, in & Y, coordinate \\
\hline $5 / 16$ & $8 / 16$ & $5 / 16$ & 0.5576 & 0.2761 \\
\hline $5 / 16$ & $8 / 16$ & $6 / 16$ & 0.483 & 0.3078 \\
\hline $5 / 16$ & $8 / 16$ & $7 / 16$ & 0.408 & 0.3392 \\
\hline $5 / 16$ & $8 / 16$ & $8 / 16$ & 0.335 & 0.2974 \\
\hline $5 / 16$ & $8 / 16$ & $9 / 16$ & 0.0931 & 0.2219 \\
\hline
\end{tabular}

Table6. Case no. 6: PBD 6/16 in and PTD 8/16 in at different ball sizes

\begin{tabular}{|c|c|c|c|c|}
\hline PBD, in & PTD, in & Ball, in & stem, in & Y, coordinate \\
\hline $6 / 16$ & $8 / 16$ & $5 / 16$ & 0.865 & 0.4233 \\
\hline $6 / 16$ & $8 / 16$ & $6 / 16$ & 0.7310 & 0.4450 \\
\hline $6 / 16$ & $8 / 16$ & $7 / 16$ & 0.598 & 0.4677 \\
\hline $6 / 16$ & $8 / 16$ & $8 / 16$ & 0.47 & 0.446 \\
\hline $6 / 16$ & $8 / 16$ & $9 / 16$ & 0.13 & 0.2588 \\
\hline
\end{tabular}

Table7. Case no. 7: PBD 6/16 in and PTD 9/16 in at different ball sizes

\begin{tabular}{|c|c|c|c|c|}
\hline PBD, in & PTD, in & Ball, in & stem, in & Y, coordinate \\
\hline $6 / 16$ & $9 / 16$ & $5 / 16$ & 0.731 & 0.3478 \\
\hline $6 / 16$ & $9 / 16$ & $6 / 16$ & 0.6505 & 0.3737 \\
\hline $6 / 16$ & $9 / 16$ & $7 / 16$ & 0.5722 & 0.4017 \\
\hline $6 / 16$ & $9 / 16$ & $8 / 16$ & 0.4948 & 0.4307 \\
\hline $6 / 16$ & $9 / 16$ & $9 / 16$ & 0.418 & 0.3758 \\
\hline
\end{tabular}

\section{RESULTS AND DISCUSSION}

The results of the partially curved and wholly curved design seats were very promising and demonstrated that the gas throughput was higher using the new designs than using the previous ones. Specifically, wholly curved design seat showed the best results among all the design seats in terms of gas flow rate.

\subsection{Pressure Profile of GLV Seat}

Following figure 5 shows the pressure distribution of the GLV for all seat types. 

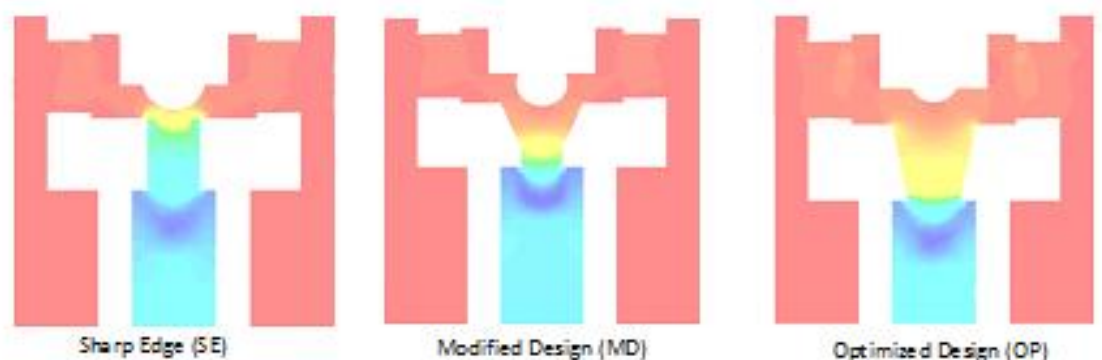

Optimised Design $\{O P\}$
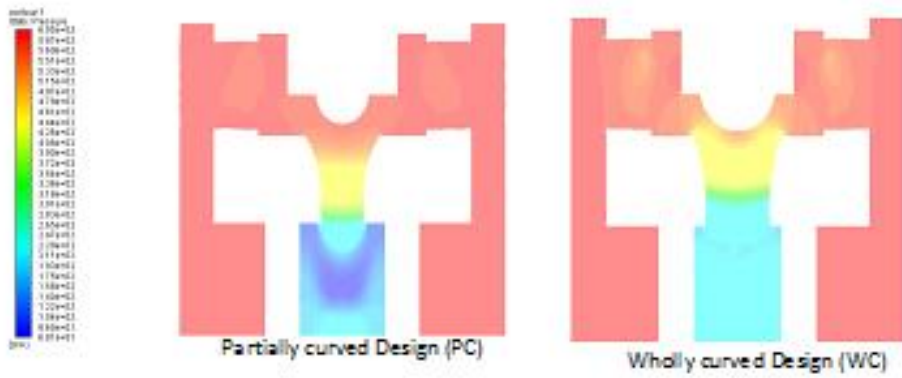

Figure5. Pressure distribution of the GLV for all seat types

Although the results show that all seat designs have a uniform pressure distribution, wholly curved design has the highest gas throughput according to the pressure profile presented in figure 5.

\subsection{Ball Size and Seat Type Optimization}

The results of the simulation work of the PC and WC seats demonstrated that the stem travel size has an inversely proportional relationship with the gas throughput. When the stem travel size was decreased by $38 \%$ (from $5 / 16$ in to $9 / 16$ in), the gas throughput was increased by $15 \%$ (fixing the PTD and PBD).

Furthermore, when the PTD was increased by 33\% (from 6/16 in to $9 / 16$ in), the gas throughput increased by $20 \%$ (fixing the stem travel and the PBD). On the other hand, the gas throughput was doubled when the PBD was increased 50\% (fixing the stem travel and the PTD).

The above results support the previous work conducted by (Kabir, 2018) when Modified and optimized seats were investigated.

The following figures present the effect of stem travel and seat sizes of MD, OP, PC and WC on the performance of GLV.

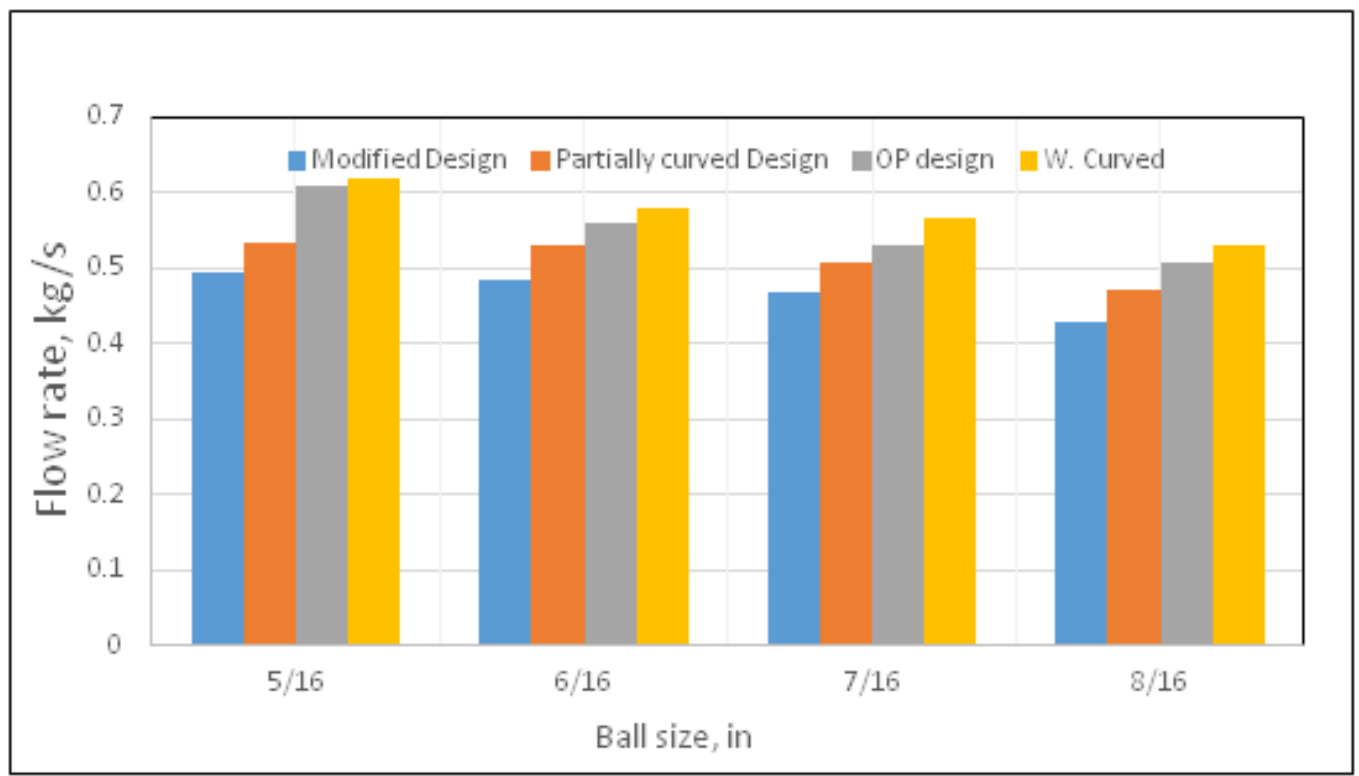

Figure6. Effect of stem travel size on the gas throughput at PBD 4/16 in and PTD 6/16 in 
Investigating Effects of the Ball Size on the Gas Throughput Using Partially Curved and Wholly Curved Seats

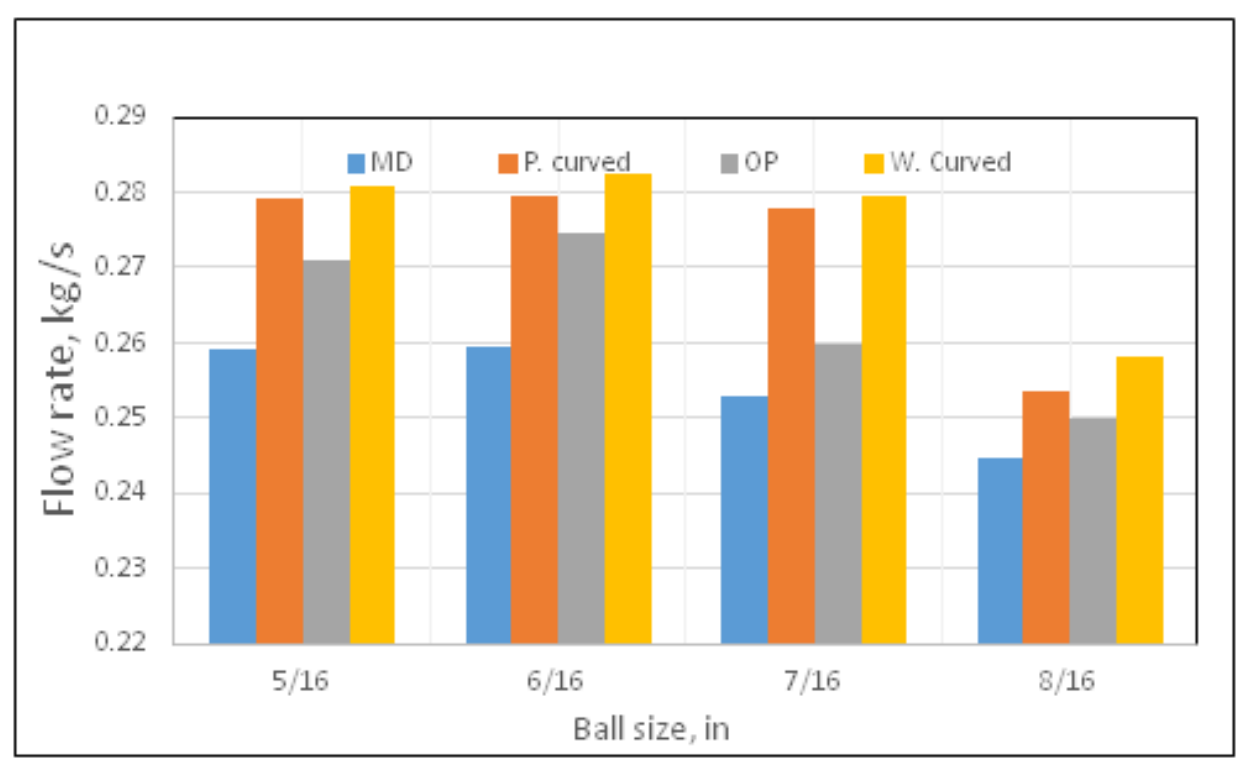

Figure7. Effect of stem travel size on the gas throughput at PBD 4/16 in and PTD 7/16 in

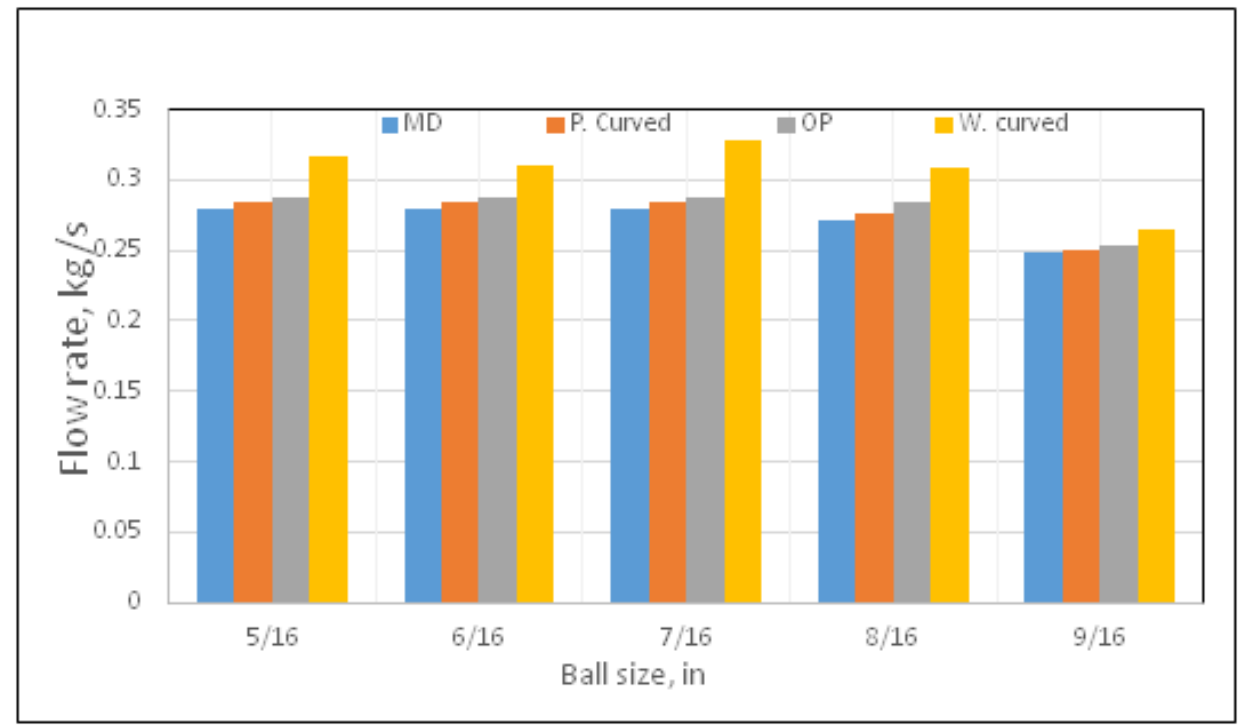

Figure8. Effect of stem travel size on the gas throughput at PBD 4/16 in and PTD 8/16 in

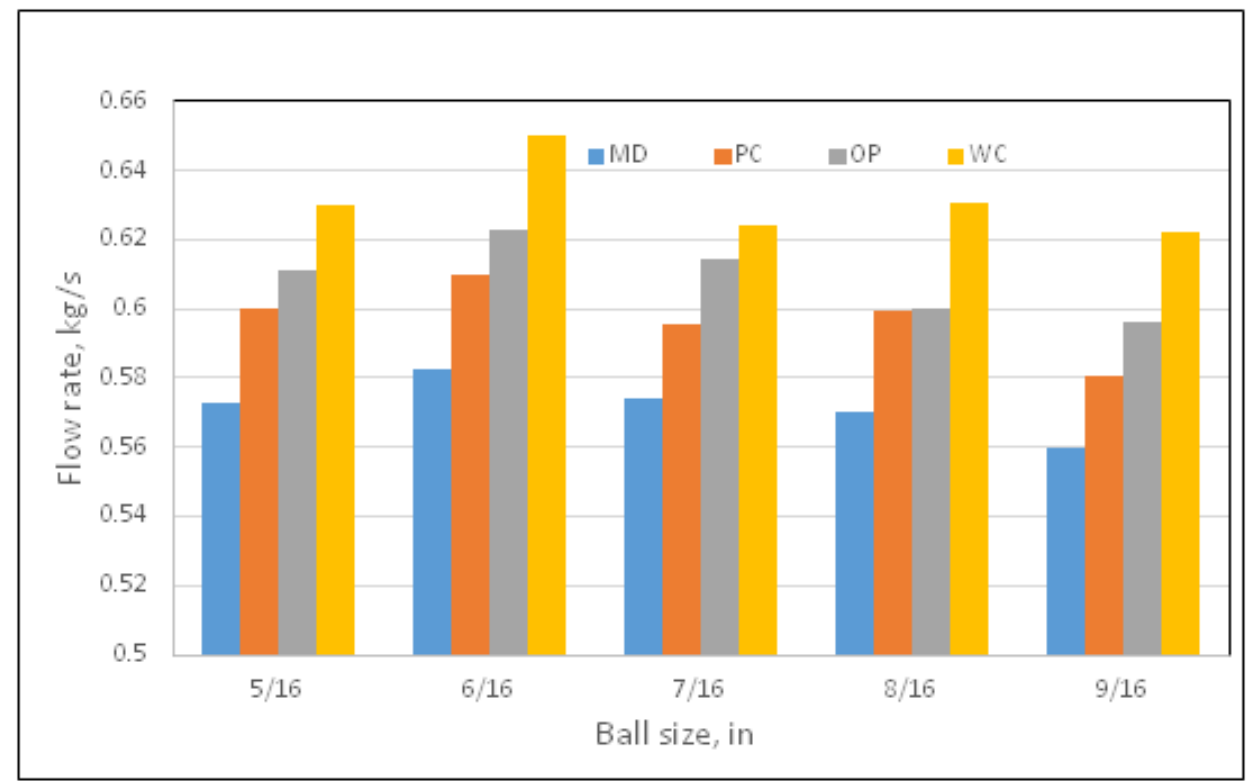

Figure9. Effect of stem travel size on the gas throughput at PBD 5/16 in and PTD 7/16 in 
Investigating Effects of the Ball Size on the Gas Throughput Using Partially Curved and Wholly Curved Seats

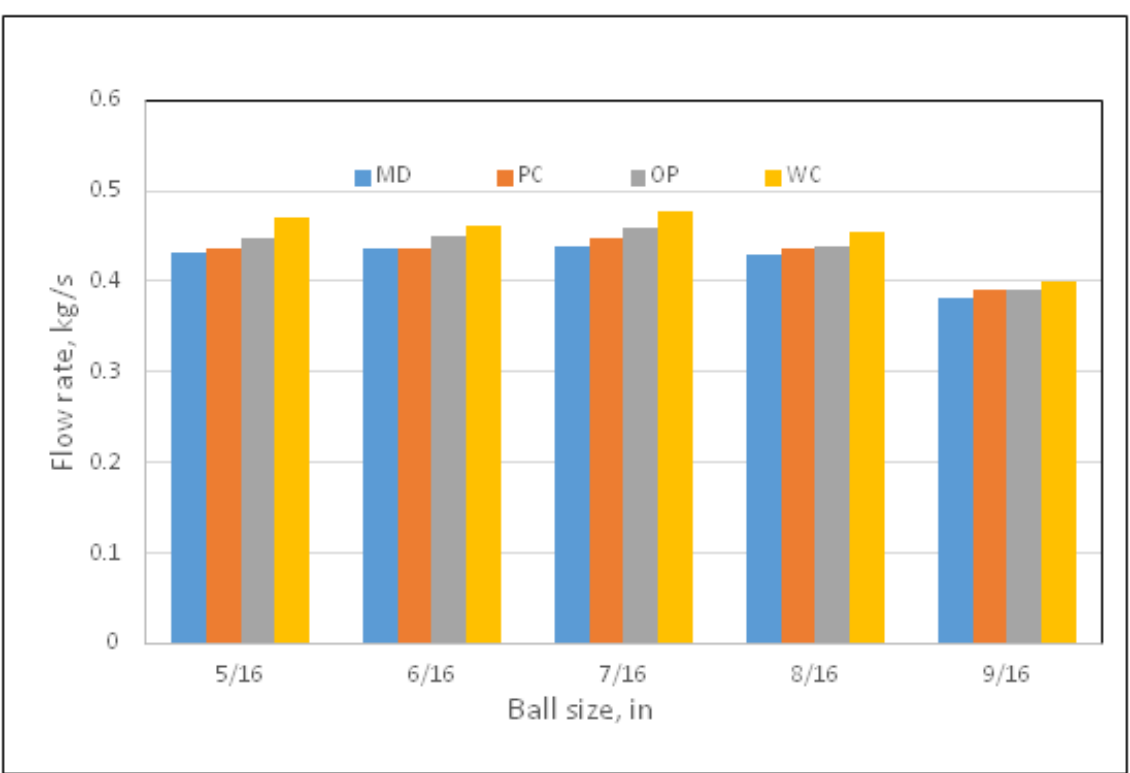

Figure10. Effect of stem travel size on the gas throughput at PBD 5/16 in and PTD 8/16 in

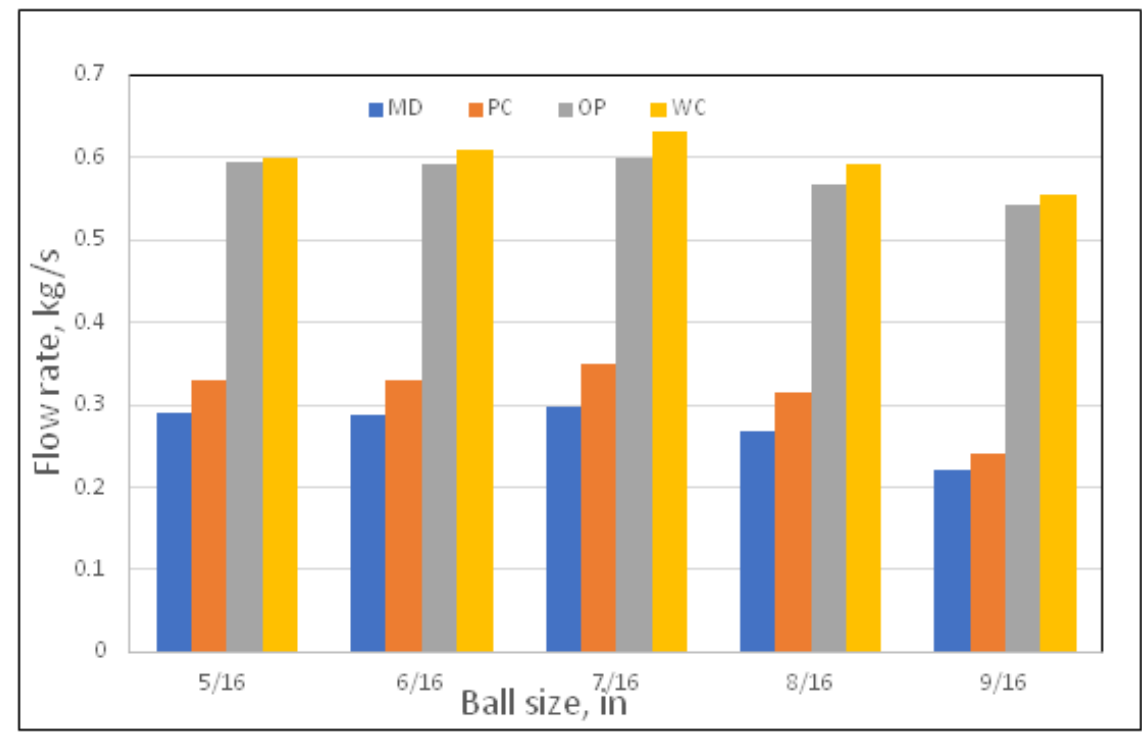

Figure11. Effect of stem travel size on the gas throughput at PBD 6/16 in and PTD 8/16 in

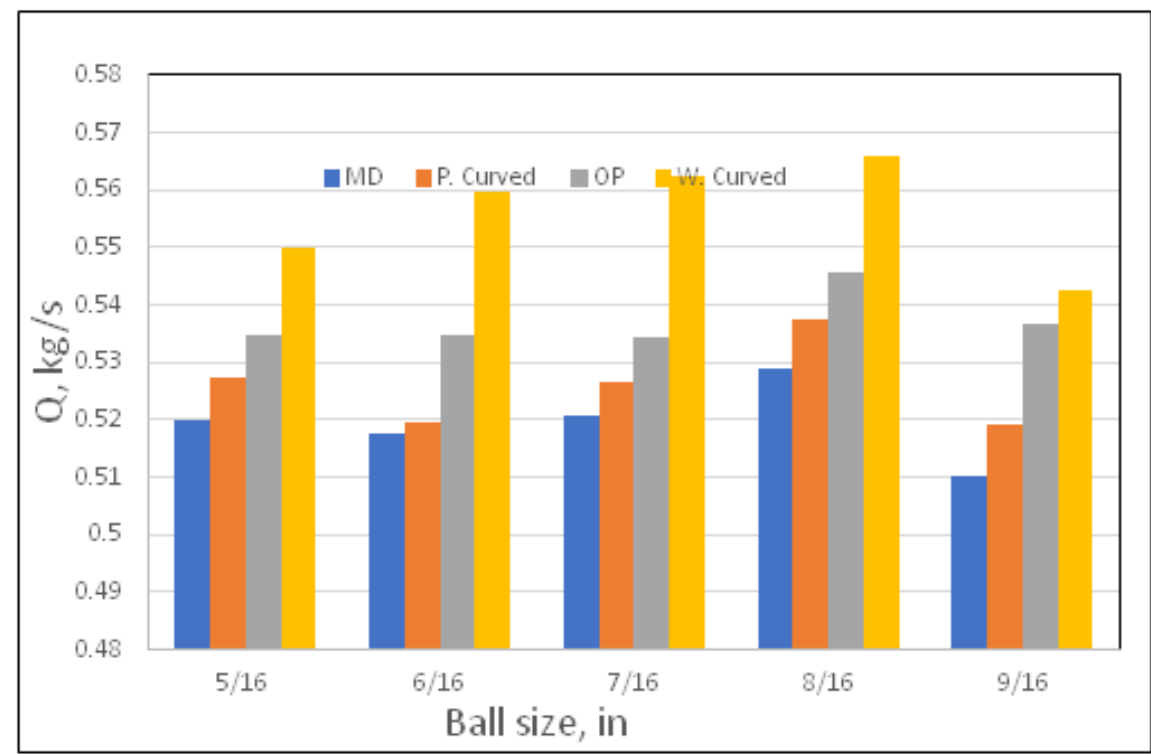

Figure12. Effect of stem travel size on the gas throughput at PBD 6/16 in and PTD 9/16 in 
Investigating Effects of the Ball Size on the Gas Throughput Using Partially Curved and Wholly Curved Seats

\subsubsection{The Best Seat Type at Various Ball Sizes}

Table 8 , table 9 , table 10 and table 11 show that the best scenario when analyzing the PBD and PTD is Wholly curved seat at various ball sizes of 5/16 in, 6/16 in, 7/16 in and 8/16 in respectively.

Table8. The best seat at Ball size 5/16 in

\begin{tabular}{|c|c|c|c|c|}
\hline PBD, in & PTD, in & Ball, in & Q, kg/s & Design \\
\hline $4 / 16$ & $6 / 16$ & $5 / 16$ & 0.619 & WC \\
\hline $4 / 16$ & $7 / 16$ & $5 / 16$ & 0.280 & W.C \\
\hline $4 / 16$ & $8 / 16$ & $5 / 16$ & 0.317 & W.C \\
\hline $5 / 16$ & $7 / 16$ & $5 / 16$ & 0.470 & W.C \\
\hline $5 / 16$ & $8 / 16$ & $5 / 16$ & 0.469 & W.C \\
\hline $6 / 16$ & $8 / 16$ & $5 / 16$ & 0.599 & W.C \\
\hline $6 / 16$ & $9 / 16$ & $5 / 16$ & 0.549 & W.C \\
\hline
\end{tabular}

Table9. The best seat at ball size $6 / 16$ in

\begin{tabular}{|c|c|c|c|c|}
\hline PBD, in & PTD, in & Ball, in & Q, kg/s & Design \\
\hline $4 / 16$ & $6 / 16$ & $6 / 16$ & 0.540 & WC \\
\hline $4 / 16$ & $7 / 16$ & $6 / 16$ & 0.282 & W.C \\
\hline $4 / 16$ & $8 / 16$ & $6 / 16$ & 0.310 & W.C \\
\hline $5 / 16$ & $7 / 16$ & $6 / 16$ & 0.650 & W.C \\
\hline $5 / 16$ & $8 / 16$ & $6 / 16$ & 0.460 & W.C \\
\hline $6 / 16$ & $8 / 16$ & $6 / 16$ & 0.610 & W.C \\
\hline $6 / 16$ & $9 / 16$ & $6 / 16$ & 0.559 & W.C \\
\hline
\end{tabular}

Table10. The best seat at ball size 7/16 in

\begin{tabular}{|c|c|c|c|c|}
\hline PBD, in & PTD, in & Ball, in & Q, kg/s & Design \\
\hline $4 / 16$ & $6 / 16$ & $7 / 16$ & 0.566 & PC \\
\hline $4 / 16$ & $7 / 16$ & $7 / 16$ & 0.279 & PC \\
\hline $4 / 16$ & $8 / 16$ & $7 / 16$ & 0.327 & WC \\
\hline $5 / 16$ & $7 / 16$ & $7 / 16$ & 0.624 & PC \\
\hline $5 / 16$ & $8 / 16$ & $7 / 16$ & 0.477 & WC \\
\hline $6 / 16$ & $8 / 16$ & $7 / 16$ & 0.639 & WC \\
\hline $6 / 16$ & $9 / 16$ & $7 / 16$ & 0.562 & W.C \\
\hline
\end{tabular}

Table11. The best seat at ball size $8 / 16$ in

\begin{tabular}{|c|c|c|c|c|}
\hline PBD, in & PTD, in & Ball, in & Q, kg/s & Design \\
\hline $4 / 16$ & $6 / 16$ & $8 / 16$ & 0.469 & PC \\
\hline $4 / 16$ & $7 / 16$ & $8 / 16$ & 0.258 & WC \\
\hline $4 / 16$ & $8 / 16$ & $8 / 16$ & 0.293 & WC \\
\hline $5 / 16$ & $7 / 16$ & $8 / 16$ & 0.409 & PC \\
\hline $5 / 16$ & $8 / 16$ & $8 / 16$ & 0.454 & WC \\
\hline $6 / 16$ & $8 / 16$ & $8 / 16$ & 0.593 & WC \\
\hline $6 / 16$ & $9 / 16$ & $8 / 16$ & 0.565 & W.C \\
\hline
\end{tabular}

Table 12 shows the best seat and stem travel size of wholly curved design which results in the highest gas throughput of $0.65 \mathrm{~kg} / \mathrm{s}$.

Table12. The best seat and stem travel size of wholly curved design

\begin{tabular}{|c|c|c|c|c|}
\hline PBD, in & PTD, in & Ball Size, in & Q, kg/s & Seat \\
\hline $4 / 16$ & $6 / 16$ & $5 / 16$ & 0.619 & W.C \\
\hline $5 / 16$ & $7 / 16$ & $6 / 16$ & 0.650 & W.C \\
\hline $6 / 16$ & $8 / 16$ & $7 / 16$ & 0.639 & W.C \\
\hline $6 / 16$ & $8 / 16$ & $8 / 16$ & 0.593 & W.C \\
\hline
\end{tabular}

Table 13 demonstrates the comparison between the wholly curved design and Sharp edge design. The new design of wholly curved was not only the best among the PC, MD and OP, but it is also was better than the Sharp edge design. The gas throughput obtained by the wholly curved design is almost twice the amount of the sharp edge design, which is currently approved and used in the oil industry.

Table13. The comparison between wholly curved design and Sharp edge design

\begin{tabular}{|c|c|c|c|c|}
\hline Seat & PBD, in & PTD, in & Ball Size, in & Q, $\mathrm{kg} / \mathrm{s}$ \\
\hline Wholly Curved & $5 / 16$ & $7 / 16$ & $6 / 16$ & 0.650 \\
\hline Sharp Edge & $5 / 16$ & $5 / 16$ & $6 / 16$ & 0.347 \\
\hline
\end{tabular}




\section{CONClusion}

In this simulation study, five main seat designs were evaluated using Computational Fluid Dynamics (CFD): The Sharp edge, modified design, the optimized design, Partially Curved Design and Wholly Curved Design. Seven seat sizes and five different ball sizes have been selected to maximize the gas throughput implementing the five designs (SE, MD, OP, PC, and WC).

- The results of the partially curved and Wholly curved design seats were very promising and demonstrated that the gas throughput was higher using the new designs than using the previous ones (SE, MD, and OP).

- Wholly curved seat is considered the best design compared to the others because it gives the highest flow rate $(0.65 \mathrm{~kg} / \mathrm{s})$.

- The results also demonstrated that all seat designs have a uniform pressure distribution, however, wholly curved design has the highest gas throughput according to the pressure profile.

- The results of the simulation work of the PC and WC seats demonstrated that the stem travel size has an inversely proportional relationship with the gas throughput. When the stem travel size was decreased by $38 \%$ (from $9 / 16$ in to $5 / 16$ in), the gas throughput was increased by $15 \%$ (fixing the PTD and PBD).

- It was also observed that there is a proportional relationship between the PTD and the gas throughput. When the PTD was increased by 33\% (from 6/16 in to 9/16 in), the gas throughput increased by $20 \%$ (fixing the stem travel and the PBD).

- Wholly curved seat design could be potentially implemented in the oil industry to improve the efficiency of the current gas lift operation and ultimately oil production.

\section{REFERENCES}

[1] Fathi Elldakli (2017) Testing, Modeling and Optimizing a Modified Design of Gas Lift Valve Seat. (Unpublished doctoral dissertation). Texas Tech University.

[2] Ehsanul Kabir, (2017), Investigating effects of ball size on the performance of gas lift valve for both modified and optimized seats, Oil \& Gas Science and Technology 73(1):15

[3] Decker, A. L., et al. (1976). Analytical methods for determining pressure response of bellows operated valves.

[4] Decker, K., et al. (1986). Computer modeling of gas-lift valve performance. In Offshore technology conference.

[5] Kulkarni, M. (2005). Gas lift valve modeling with orifice effects. (Unpublished master's thesis). Texas Tech University., Lubbock

[6] Shahri, M. A. (2011). Simplified and rapid method for determining flow characteristics of every gas-lift valve (glv) (Unpublished doctoral dissertation). Texas Tech University.

[7] Winkler, H., Camp, G., et al. (1987). Dynamic performance testing of single-element unbalanced gas-lift valves. SPE Production Engineering, 2(03), 183-190.

[8] Brown, K. 1973. Gas Lift Theory and Practice. P.202

[9] Winkler, W., \& Blann, J. (2007). Petroleum engineering handbook, chapter 11. Society of Petroleum Engineers.

[10] Aliyeva, F., \& Novruzaliyev, B. 2015. Gas Lift - Fast and Furious. In proceedings of the SPE Annual Caspian Technical Conference \& Exhibition.

[11] King, W. (1944, January 18). Time and volume control for gas. Google Patents. (US Patent 2,339,487).

[12] Xu, Z., Richard, B. M., \& Kritzler, J. H. (2013, September 30). Smart Gas Lift Valves Enhance Operation Efficiency of Offshore Wells. In proceedings of the Annual Technical Conference \& Exhibition.

Citation: Saleh Ahmed, et.al, (2019). "Investigating Effects of the Ball Size on the Gas Throughput Using Partially Curved and Wholly Curved Seats", International Journal of Petroleum and Petrochemical Engineering (IJPPE), 5(3), pp.1-9, DOI: http://dx.doi.org/10.20431/2454-7980.0503001

Copyright: $\odot 2019$ Authors. This is an open-access article distributed under the terms of the Creative Commons Attribution License, which permits unrestricted use, distribution, and reproduction in any medium, provided the original author and source are credited 